\title{
Image Segmentation Through Dual Pyramid of Agents
}

\author{
K. Idir, H. Merouani, and Y. Tlili \\ Laboratory of computer science Research., Pattern Recognition Group, \\ Dept. of computer science - Faculty of engineer science, \\ Badji Mokhtar University, BP.12-23200, Annaba, Algeria \\ Karima_id2@yahoo.fr, hayet_merouani@yahoo.fr, guiyam@yahoo.fr
}

\begin{abstract}
An effective method for the early detection of breast cancer is the mammographic screening. One of the most important signs of early breast cancer is the presence of microcalcifications. For the detection of microcalcification in a mammography image, we propose to conceive a multiagent system based on a dual irregular pyramid.

An initial segmentation is obtained by an incremental approach; the result represents level zero of the pyramid. The edge information obtained by application of the Canny filter is taken into account to affine the segmentation. The edge-agents and region-agents cooper level by level of the pyramid by exploiting its various characteristics to provide the segmentation process convergence.
\end{abstract}

Keywords: Dual Pyramid, Image Segmentation, Multi-agent System, Region/Edge Cooperation.

\section{Introduction}

Image segmentation is one of the most widely used steps in the process of reducing images to get useful information. It allows the partitioning of image into several distinct regions.

We propose for the detection of microcalcification in a mammography image, to cooperate region-agents obtained by an incremental approach and edge-agents obtained by a Canny filter in a pyramidal structure.

Initially, a short outline of the irregular pyramids is presented, then we expose in brief way some work carried out in image segmentation by multi-agent approach, there after we present our approach while arguing the choice of the dual pyramid and the role of the agents. Finally we conclude this paper by some prospects for possible improvements.

\section{Irregular Pyramid}

A pyramid is a stack of images with decreasing resolutions; the bottom level of a pyramid is the original image. Each level of the pyramid can be described by the neighborhood graph where the set of vertices correspond to regions of the level and 
the edges' set correspond to the adjacency relations between regions. The algorithm for the pyramid construction proceeds in the following steps [1]:

- Creation of level zero (base), starting from image to be treated.

- Construction of the similarity graph starting from the adjacency graph. In order to hold account of the contents of image, each vertex is connected only to vertices, which are declared to him similar.

- The graph of the $\mathrm{k}$ level is obtained by processing decimation on the graph of $\mathrm{k}-1$ level. This decimation procedure is based on two rules.

1. Two neighbors at a given level cannot both survive at the next level;

2. For each non-surviving cell, there is at least one surviving cell in its neighborhood.

The decimation can be:

- Stochastic: [11] the choice of the surviving vertices is done randomly.

- Adaptative: [9] a vertex survives if it locally minimizes an interest operator based on the variance of the region associated to the vertex.

- Assignment of each non survivor vertex to one survivor parent.

- Attributes of each surviving vertex are calculated according to the attributes of vertices of the level below that represent.

- Construction of the neighborhood graph of the new level.

The irregular pyramids operate on general graph structures instead of the regular neighborhoods as in the case of regular pyramids (example: "Quadtree"). The cells of one level represent each one a region of which the form is not constrained by a geometrical pattern (square, triangle, polygon). The result obtained by the application of the irregular pyramids is independent of the course of image.

However these pyramidal structures can generate a non-existent borders or forget existing discontinuities, to avoid this disadvantage, cooperative approaches are proposed. For a natural aspect of cooperation between region and edge multi-agent systems are well adapted to develop such approaches.

\section{Multi-agent Approaches in Image Segmentation}

According to the application developed in previous works, different kind of agents with a variety of characteristics, interaction and coordination concepts can be found.

One of the aspects of Boucher system [2] is the manner of launching agents in the next image of the sequence according to what is being held in current image. The agents thus launched start by segmenting their components and will meet progressively while growing. Two agents which meet and discover that work both on the same component of image can fuse their efforts in order to decrease the number of redundant agent. The agents used for the study of cytological images sequences, have four behaviors: The perception behavior to evaluate pixels, the interaction behavior to manage fusions, the differentiation behavior to evaluate the primitive in order to determinate the kind of component is closed to and the reproduction behavior to create agents at certain places of image.

In a context of MRI segmentation, Germond [7] cooper edge-agents based on ( $\left.A^{*}\right)$ algorithm and region-growing agents specialized for the detection of the white matter 
(WM) and the grey matter (GM) of the brain. The system comprises a deformable model that provides valuable information on the brain boundary, to position the GMagents that allow eventually the localization of WM-agents. Edge-agents are used to refine brain boundary assessed by the deformable model.

Duchesnay [5] presents a society of agents (region-agent, edge-agent) organized in irregular pyramid while detailing the initialization process of the pyramid and the total control which intervenes in the passage of one level to another. An agent of the pyramid stands for a region primitive (obtained by the Quadtree) method or edge primitive, he will supplement this primitive while merging with others agents by running a sequence of seven behaviors:

1. Territory marking and features extraction: each agent marks a territory that corresponds to its primitive

2. Exploration: Agents are exploring a shared environment around their territory to discover their neighbors.

3. Merging planning: each region agent tries to find out (similar) neighbors to merge with.

4. Cooperation: agents cooperate with each other to enhance the quality of their merging plan.

5. Decimation: the number of agents has to decrease level by level, then a distributed decimation protocol, performed by the agents, selects survivors.

6. Reproduction: each survivor agent creates a new agent in the next level of the pyramid.

7. Attachment: all agents should be represented in the next level, then each nonsurviving agent looks for the best representative in its neighborhood.

Region-agents and edge-agents cooperate for the segmentation of MRI, in a multiagent plate forms proposed by Settache [12]. Two images are inputted to the multiagent system, region-chart (obtained by Quadtree algorithm) and edge-chart (obtained by a Deriche filter, then chaining of edges), from which region and edge agents are launched. Segmentation-agents, whose behaviors are defined by an automat related on the region-agents and the edge-agents, are responsible for the improvement of the initial pre-segmentation.

\section{Proposed Approach}

We propose to conceive a multi-agent system for image segmentation, which allows a cooperation region/edge, and it differs primarily from the work presented in Sect. 3 by the use of dual pyramid.

\subsection{Region Map}

The growing region method (pixel aggregation process) is chosen for pre-segmenting the image into regions. This approach preserves the form of each region. To build a "region" primitive, first select a special set of pixels called seeds. Regions are grown by aggregating to the seeds pixels which verify a fixed criterion. Thus, a membership of a pixel into region taking into account: information of region and local information relating to the pixel. 
The evaluation of a pixel to annex to a region depends on two weighted homogeneity criteria: average of gray level and the variance of region. The image result is the base of our pyramid, where each region will be represented by an agent.

\subsection{Edge Map}

To affine segmentation, we propose to hold account the edge information, obtained by applying the Canny filter [3].

\subsection{The Dual Pyramid}

The use of simple graphs to the irregular pyramids forces to have only one edge between two vertices in the graph, thus, it is unable to distinguish from the graph an adjacency relation from inclusion relation between two regions. This limitation was raised by Kropatsch [10], Willersinn [13] and others who propose to build a dual irregular pyramid complementing each level of an irregular pyramid by its dual graph during construction. The dual graph preserves the topology of the adjacencies and correctly codes the inclusion relation between regions.

The basic process for construction of the dual pyramid is the dual decimation.

Decimation Parameters. $G_{i}\left(V_{i}, E_{i}\right)$ a planar graph, $S_{i} \subset V_{i}$ a subset of surviving vertices and $N_{i, i+1} \subset E_{i}$ a subset of primary non-surviving edges. The couple $\left(S_{i}, N_{i, i+1}\right)$ called decimation parameters determine the structure of an irregular pyramid. Every non-surviving vertex (Child) must be connected to one surviving vertex (Parent) in a unique way. The selection of the surviving vertices can be done according to rules of decimation mentioned in Sect. 2.

Dual Decimation. Dual decimation combines the selection of decimation parameters and the dual contraction that proceeds in two basic steps dual edge contraction and dual face contraction. (Fig. 1)

1. Selection of the decimation parameters $\left(S_{i}, N_{i, i+1}\right)$ :

Identification of the survivors and non-survivors.

2. Dual edge contraction $\mathrm{N}_{\mathrm{i}, \mathrm{i}+1}$ :

The contraction of a primary non-surviving edge from $\mathrm{N}_{\mathrm{i}, \mathrm{i}+1}$, consists in the identification of its endpoints (vertices) and the removal of both the contracted edge and its dual edge.

After dual contraction, faces with degree one or two may result. They correspond to self-loop and double edges in the neighborhood graph.

A second (dual) contraction process 'cleans' the dual graph from such degenerated faces but not those enclosing any surviving parts of the graph. They are necessary to preserve correct structure.

\section{Dual face contraction:}

- Selection of the decimation parameters $\left(\mathrm{S}_{\mathrm{i}}, \mathrm{N} *_{\mathrm{i}, \mathrm{i}+1}\right)$ :

- $S^{*}$, the set of faces with degree above 2.(surviving faces)

- $\mathrm{N}_{\mathrm{i}, \mathrm{i}+1}$ denotes the set of edges having one surviving faces as one end vertex and non-surviving faces as the other end vertex. 
- Dually contract edge $N^{*}, i+1$

- Repeat until all degenerated faces have been eliminated.

The relation between two pairs of dual graphs, $\left(\mathrm{G}_{\mathrm{i}}, \overline{G_{i}}\right)$ and $\left(\mathrm{G}_{\mathrm{i}+1}, \overline{G_{i+1}}\right)$ established by dual graph contraction with decimation parameters $\left(S_{i}, N_{i, i+1}\right)$ is expressed by function $\mathrm{C}$

$$
\left(G_{i+1}, \overline{G_{i+1}}\right)=C\left[\left(G_{i}, \overline{G_{i}}\right),\left(S_{i}, N_{i, i+1}\right)\right]
$$

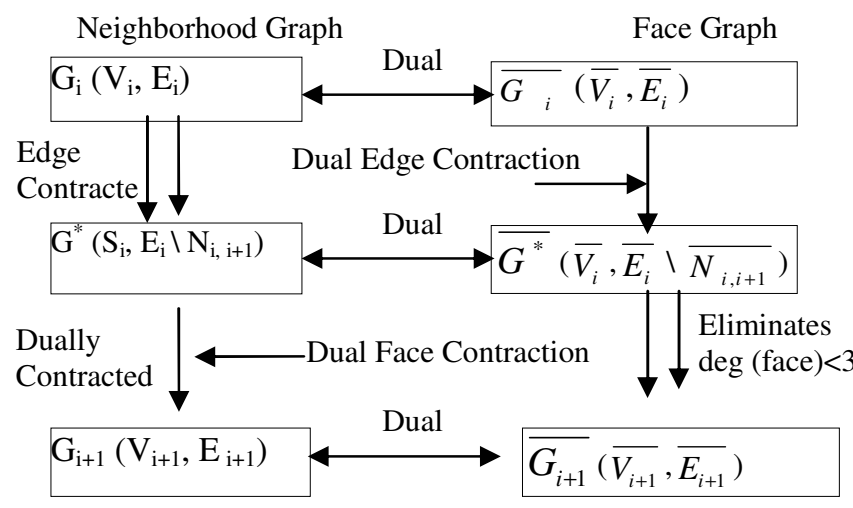

Fig. 1. Dual Graph Contraction: (Extract from ([8]) $\left(\mathrm{G}_{\mathrm{i}+1}, \overline{G_{i+1}}\right)=\mathrm{C}\left[\left(\mathrm{G}_{\mathrm{i}}, \overline{G_{i}}\right),\left(\mathrm{S}_{\mathrm{i}}, \mathrm{N}_{\mathrm{i}, \mathrm{i}+1}\right)\right]$

\subsection{Dual Pyramid of Agent for the Detection of Microcalcification}

An effective method for the early detection of breast cancer is the mammographic screening. One of the most important signs of early breast cancer is the presence of microcalcifications. A microcalcification is a tiny white speck seen on a mammogram. It represents flecks of calcium salts, and is often the only indicators of malignant tumors [6].

For the detection of microcalcification in a mammography image, we propose to conceive a multi-agent system based on pyramidal structure.

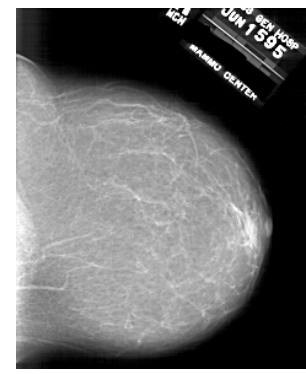

(a) Initial Image.

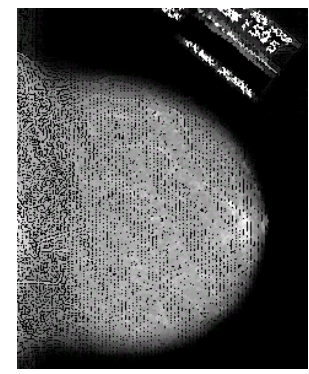

(b) Region map

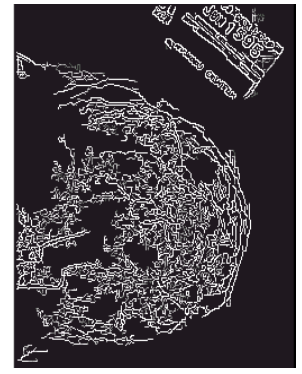

(c) Edge map

Fig. 2. Preprocessing of the image (Extract from [4]) 
The use of the agents instead of simple vertices enriches the decisional framework of the approach. Indeed the agents provide a concept particularly adapted for the expression of the cooperation and the negotiation between closed regions.

Different kinds of agent are proposed for our system:

- Agents allowing the control and the correct working of the multi-agents system: user interfaces-agents, monitor-agent, sequencer-agent and agent known as dualagent to control the various steps of the dual decimation.

- A region-agents and edge-agents: Each agent is related to a primitive region from region-map (figure $2 b$ ) or edge from edge-map (figure $2 \mathrm{c}$ ). It has all relevant information about it (examples: average and compactness of the region, length and continuity of edge).

- The interaction for the construction of the pyramid allows two agents provided with certain behaviors, to join together their primitives (region or edge):

- A behavior allowing agent to mark a territory corresponding to its primitive (region, edge).

- A behavior allowing agent to discover its neighbors. The connection of region agent is restricted to 4 neighbors, to have a planar graph at the base level of the pyramid. For the neighborhoods of the edge-agent we use the distance between the ends of edge.

- A behavior allowing agent to interact, to decide who survive and with which agent he wish to merge by calculating a fusion desire of his neighborhoods expressed in function of the average of grey level and the variance of regions. A candidate regions list of a region-agent represents regions with fusion desire greater than a fixed threshold.

- A behavior allowing agent to confirm a fusion, for example region-agent asks an edge-agent about an edge between two candidates regions to fuse.

- A behavior to select the survivor's agents by application of the rules of an adaptative decimation where the interest operator of an agent is the sum of the desire of fusion for all his candidates regions.

- A behavior allowing each non-survivor agent to be represented by the best survivor agent in its neighbors at the next level of the pyramid

- A behavior allowing a surviving agent to create a new agent at the next level of the pyramid.

To control the process of the dual decimation a dual-agent executes two behaviors:

- A behavior allowing to build a dual graph, to extract faces, discover neighborhood of faces, find correspondence between dual graph and adjacency graph.

- A behavior to control the process, relating to the dual contraction of edges and faces.

\section{Conclusion}

The image segmentation constitutes the base of the process image interpretation; it remains an active subject of research in artificial vision. Many techniques are 
available to deal with the image segmentation problems. In this article, we propose to cooperate region based-method and edge based-method by multi-agent system in a dual pyramid for segmenting mammography image.

This work is in progress, we envisage to implement various modes of cooperation: cooperation region/edge, region/region and edge/edge in order to ensure the convergence of the segmentation process.

\section{References}

1. P. Bertolino. Contributions des pyramides irrégulières en segmentation d'images multirésolution. Ph.D. thesis, Institut National Polytechnique de Grenoble, 1995

2. Boucher. Une approche décentralisée et adaptative de la gestion d'informations en vision, Application à l'interprétation d'images de cellules en mouvement. Ph.D. thesis, Université Joseph Fourier, Grenoble, 1999.

3. J.F. Canny. A computational approach to edge detection. Patt Ana.Mach. Int, 8(6):679698, 1986.

4. DDSM: Digital Database for Screening Mammography. University of South Florida. http://marathon.csee.usf.edu/Mammography/Database.html00

5. E. Duchesnay. Agents situés dans l'image et organisés en pyramide irrégulière. Contribution à la segmentation par une approche d'agrégation coopérative et adaptative. Ph.D. Université de Rennes-1, 2001.

6. F. Gaspoz. Mammographie digitale \& Analyse d'images par ordinateur. Laboratoire TIMC - IMAG. Faculté de Médecine Grenoble. Université Joseph Fourier Grenoble, France. 2003

7. L. Germond, M.Dojat, C. Taylor, C. Garbay. A Cooperative Framework for Segmentation of MRI Brain Scans. Artif. Intell. in Med. 20 (2000) 277-94.

8. Y. Haxhimusa, W.G. Kropatsch. Hierarchical Image Partitioning with Dual Graph Contraction. Technical Report PRIP-TR-81, Institute of Computer Aided Automation 183/2, Patt. Recogn. Image. Proc Group, Austria, 2003

9. J.M. Jolion and A. Montanvert. The adapted pyramid: a framework for $2 \mathrm{~d}$ image analysis. Computer Vision Graphics and Image Processing, 55(3):339-348, May 1992

10. W.G. Kropatsch. Building irregular pyramids by Dual Graph Contraction. Technical Report PRIP-TR-35, Institute of Automation 183/2, Dept. for Patt. Rec. Image. Proc, TU Wien,Austria, 1994

11. P. Meer. Stochastic image pyramids. Comp. Vision. Graph. Image Proc.. 45 (3): 269-294. 1989.

12. H. Settache. Une plate-forme multi-agents pour la segmentation d'images: Application dans le domaine des IRM cérébrales 2D. DEA Report, Université de Caen, 2002

13. D. Willersinn. Parallel Graph Contraction for Dual Irregular Pyramids. PRIP-TR 28, Institute for Automation, 183/2, Technical University of Vienna, Austria, 1994 